June 1992

\title{
Neuroleptic Malignant Syndrome: Diagnostic Dilemma in the Medically III
}

\author{
Mahmoud A. Parsa, M.D. \\ University Hospitals, Cleveland, Ohio \\ Andrew J. Picken, \\ University Hospitals, Cleveland, Ohio \\ Robert Keating, M.D. \\ University Hospitals, Cleveland, Ohio
}

Follow this and additional works at: https://jdc.jefferson.edu/jeffjpsychiatry

Part of the Psychiatry Commons

Let us know how access to this document benefits you

\section{Recommended Citation}

Parsa, M.D., Mahmoud A.; Picken,, Andrew J.; and Keating, M.D., Robert (1992) "Neuroleptic Malignant Syndrome: Diagnostic Dilemma in the Medically III," Jefferson Journal of Psychiatry. Vol. 10 : Iss. 2 , Article 6.

DOI: https://doi.org/10.29046/JJP.010.2.004

Available at: https://jdc.jefferson.edu/jeffjpsychiatry/vol10/iss2/6

This Article is brought to you for free and open access by the Jefferson Digital Commons. The Jefferson Digital Commons is a service of Thomas Jefferson University's Center for Teaching and Learning (CTL). The Commons is a showcase for Jefferson books and journals, peer-reviewed scholarly publications, unique historical collections from the University archives, and teaching tools. The Jefferson Digital Commons allows researchers and interested readers anywhere in the world to learn about and keep up to date with Jefferson scholarship. This article has been accepted for inclusion in Jefferson Journal of Psychiatry by an authorized administrator of the Jefferson Digital Commons. For more information, please contact: JeffersonDigitalCommons@jefferson.edu. 


\title{
Neuroleptic Malignant Syndrome: Diagnostic Dilemma in the Medically Ill
}

\author{
Mahmoud A. Parsa, M.D., \\ Andrew J. Picken \\ and Robert Keating, M.D.
}

\begin{abstract}
Neuroleptic malignant syndrome is a potentially fatal complication of neuroleptic treatment. It is characterized by a variety of signs and symptoms associated with autonomic instability, and hypothalamic and extrapyramidal dysfunction. Since other medical disorders may mimic its clinical presentation, the accurate recognition of this syndrome in the medical setting is often a diagnostic dilemma. Three medically ill patients with a clinical presentation suggestive of neuroleptic malignant syndrome are presented here. The authors discuss the complexity of making the diagnosis, and offer a brief review of the literature.
\end{abstract}

Since it was first described in 1960, approximately 1000 cases of neuroleptic malignant syndrome (NMS) have been reported in the literature (1-2). Although the precise pathophysiology is not well understood, NMS is widely recognized as a potentially fatal complication of treatment with neuroleptic agents. Studies suggest that it is related to decreased central dopaminergic activity, most commonly precipitated by dopamine D2 receptor blockade in the striatal and hypothalamic systems (3).

Experts have not been able to identify accurately a subpopulation at increased risk for developing the syndrome. However, a genetic component of NMS vulnerability has been hypothesized, and a recent familial case report proposes that NMS susceptibility may be, in part, a heritable characteristic (4). Case reviews suggest that risk factors for developing NMS include: 1) previous history of NMS, 2) intramuscular delivery and/or abrupt increase in rate or total dosage of neuroleptic agent, 3) antecedent dopamine agonist or anticholinergic medication withdrawal, 4) concurrent use of other psychotropic drugs, 5) ethanol abuse or dependence, 6) pre-existing brain pathology, 7) concomitant medical or neurological illness, 8) dehydration, and 9) psychomotor agitation (5-7). Gender ratio of NMS is approximately 2:1 male:

Mahmoud A. Parsa, M.D., Fellow, Department of Psychiatry, University Hospitals of Cleveland, Case Western Reserve University, Cleveland, Ohio.

Andrew J. Picken, Senior Medical Student, Case Western Reserve University, Cleveland, Ohio. Robert Keating, M.D., Resident, Department of Family Medicine, Fairview Hospital, Cleveland, Ohio. 
female, with an average age of onset of 38-40. Risk factors for NMS are summarized in Table 1.

Diagnosis of NMS requires differentiation from clinically similar syndromes including the serotonin syndrome (8-9), monamine oxidase inhibitor-induced hypertensive crisis, malignant hyperthermia, heat stroke, and lethal catatonia (10-11). Various medical and neurological illnesses may also resemble NMS, therefore, differential diagnosis of this syndrome in a medical setting may be problematic (12). Investigators have outlined diagnostic criteria to assist in distinguishing NMS from other clinically similar entities $(7,12)$. The major criteria are based on the presence of 1) autonomic instability, 2) extrapyramidal dysfunction, 3) hyperthermia, and 4) clouded consciousness. In addition, a variety of abnormal laboratory values have been outlined as minor criteria; 1) elevated serum creatine kinase (CK) activity over 1000 U/liter, 2) white blood cell count (WBC) over $15.0 \times 10^{9} \mathrm{E} /$ liter, 3) elevated liver enzymes, 4) myoglobinuria, 5) electrolyte abnormalities, and 6) diffuse slowing on electroencephalogram (13). Diagnostic criteria for NMS are summarized in Tables 2 and 3 .

Treatment for NMS includes prompt recognition of the syndrome, immediate

TABLE 1.

Application of Risk Factors for NMS

\begin{tabular}{lccc}
\hline & Case \#1 & Case \#2 & Case \#3 \\
\hline Demographic & & & \\
Sex & $\mathrm{M}$ & $\mathrm{M}$ & $\mathrm{M}$ \\
Age & 67 & $\mathrm{MR} / \mathrm{CS}$ & $\mathrm{OBS}$ \\
Psychiatric Diagnosis & $\mathrm{MR}$ & & \\
Neuroleptic Related & & 2.5 & 5 \\
Maximum dose (mg/24 hours) & 7 & no & yes \\
Initiated/Increased dose & yes & high & high \\
Potency & high & oral & oral \\
Route & IM & yes & no \\
Polypharmacy (psychotropics) & yes & & \\
Medical/Neurological Status & & no & yes \\
Psychomotor agitation & yes & yes & yes \\
Concurrent illnesses & yes & yes & yes \\
Preexisting brain pathology & yes & no & no \\
Alcohol/Substance abuse & no & no & no \\
Dehydration & yes & ? & no \\
Previous NMS & ? & no & no \\
Iron deficiency anemia ${ }^{2}$ & yes & &
\end{tabular}

${ }^{1}$ Case \# 1 received a combination of trifluperazine $10 \mathrm{mg}$ and haloperidol $14 \mathrm{mg}$ over a period of 48 hours.

${ }^{2}$ Although controversial, low serum iron has been reported as a potential risk factor for NMS (18).

Abbreviations: IM; intramuscular-MR; mental retardation-CS; chronic schizophreniaOBS; organic brain syndrome. 
discontinuation of psychotropic medications, and institution of supportive therapy. Dopaminergic agonists such as bromocriptine, amantadine, L-dopa, and calcium channel blockers, such as dantrolene sodium, have demonstrated effectiveness in resolution of the symptoms, and reduction in associated mortality (14). A mortality rate of $20-30 \%$ secondary to complications (pulmonary, renal, cardiovascular) has been reported in untreated cases (15).

We report three patients who presented to the medical service with fever, and unexplained elevated serum CK. Following psychiatric consultation, one case was diagnosed with NMS, and the possibility of NMS was ruled out in another. Assessment for NMS in the third patient was difficult due to a complex medical presentation.

\section{CASE \# 1}

A 67-year-old man was transferred from a nursing home to University Hospitals of Cleveland for evaluation and treatment of a right lower extremity fracture. The patient's past medical history was significant for gastrointestinal bleeding and iron deficiency anemia. His psychiatric history was remarkable for mental retardation secondary to a febrile illness at age four. Before admission the patient had been receiving furosemide $20 \mathrm{mg}$ daily, iron sulfate 300 $\mathrm{mg}$ twice daily. His treatment also included nortriptyline $25 \mathrm{mg}$ twice daily and trifluperazine 2 $\mathrm{mg}$ twice daily for psychological and behavioral abnormalities.

On day three of hospitalization, following an orthopedic procedure, the patient demonstrated increasing agitation, and haloperidol in a dosage of $2 \mathrm{mg}$ oral or intramuscular every 4 hours as needed, and benztropine were added to his admission medications. Over the next two days, he received a total of $14 \mathrm{mg}$ intramuscular haloperidol and $2 \mathrm{mg}$ intramuscular trifluperazine in addition to his standing regimen. Subsequently, the patient developed motor

TABLE 2.

Application of Keck's Diagnostic Criteria of NMS

\begin{tabular}{lccc}
\hline & Case \#1 & Case \#2 & Case \#3 \\
\hline Major Criteria & & & \\
Hyperthermia $\left(>=38^{\circ} \mathrm{C}\right)$ & yes & yes & yes \\
Extrapyramidal Dysfunction & yes & no & yes \\
Autonomic Dysfunction ${ }^{2}$ & yes & no & yes \\
Minor Criteria $^{3}$ & & & \\
Clouded Consciousness $_{\text {Serum CK }>1000 \mathrm{U} / \text { liter }}$ & yes & no & yes \\
WBC $>15.0 \times 109 \mathrm{E} /$ liter & yes & yes & yes \\
\hline
\end{tabular}

${ }^{1}$ Two or more of the following: muscular rigidity; dystonic reaction; cogwheeling; parkinsonism.

${ }^{2}$ Two or more of the following: fluctuating blood pressure; tachycardia; tachypnea; diaphoresis; urinary incontinence.

${ }^{3}$ In the absence of one of the three major criteria, probable diagnosis is still permitted if the patient clearly meets the two remaining major criteria, and displays one of the minor criteria. 
TABLE 3.

Application of Caroff's Diagnostic Criteria of NMS

\begin{tabular}{lccc}
\hline & Case \#1 & Case \#2 & Case \#3 \\
\hline $\begin{array}{l}\text { Neuroleptic treatment within } 7 \text { days of onset }(2-4 \\
\text { weeks for depot preparations) }\end{array}$ & yes & yes & yes \\
Hyperthermia $\left(>=38^{\circ} \mathrm{C}\right)$ & yes & yes & yes \\
Muscle rigidity & yes & no & yes \\
Five of the following & & & \\
Altered mental status & yes & yes & yes \\
Tachycardia & yes & no & yes \\
Hyper/hypotension & yes & no & yes \\
Tachypnea/Hypoxia & yes & no & no \\
Diaphoresis/Sialorrhea & yes & no & yes \\
Dysarthria/Dysphagia & yes & no & ? \\
Tremor & yes & yes & no \\
Incontinence & no & no & no \\
CK elevation & yes & yes & yes \\
Myoglobinuria & no & no & no \\
Leukocytosis & yes & yes & no \\
Absence of other etiologies & yes & no & no \\
\hline
\end{tabular}

dysfunction, aspiration pneumonia, and became increasingly confused. Antibiotic therapy was initiated to treat the pneumonia.

At psychiatric consultation the patient was diaphoretic, with an oral temperature of $38.9^{\circ} \mathrm{C}$, pulse of $104 / \mathrm{min}$, respiration of $32 / \mathrm{min}$, and fluctuating blood pressure between $160 / 90$ to $108 / 50 \mathrm{mmHg}$. Mental status examination revealed confusion, disorientation, and an inability to communicate verbally. Neurological examination demonstrated marked extrapyramidal signs, including increased muscle tone, with diffuse tremor, cogwheeling, and lead-pipe rigidity in the upper extremities. Serum chemistries revealed a CK level of 1267 $\mathrm{U} /$ liter, leukocytosis $\left(15.0 \times 10^{9} \mathrm{E} /\right.$ liter $)$, and normal electrolytes. A tentative diagnosis of NMS was made. All psychotropic medications were discontinued, supportive therapy was initiated, and oral bromocryptine $2.5 \mathrm{mg}$ three times daily was initiated along with supportive therapy.

Over the following four days the patient's mental status cleared, extrapyramidal symptoms resolved, and vital signs stabilized (temperature $36.6^{\circ} \mathrm{C}$, pulse $72 / \mathrm{min}$, respiration $12 / \mathrm{min}$, and blood pressure $120 / 70 \mathrm{mmHg}$ ). Laboratory values normalized, with a CK of 123 $\mathrm{U} /$ liter, and WBC of $7.70 \times 10^{9} \mathrm{E} /$ liter. Bromocriptine was discontinued, and following a full course of antibiotic therapy for pneumonia the patient was discharged in improved condition.

\section{CASE \#2}

A 72 year old man with a preadmission diagnosis of chronic schizophrenia and mental retardation was hospitalized for evaluation and treatment of weakness, lethargy, and fever of unknown origin. His past medical history was significant for parkinsonism, hypertension, emphysema, with chronic bronchitis, and a hearing deficit. Past surgical history included a transurethral prostatectomy, and cholecystectomy. Medications on admission included verapamil $240 \mathrm{mg}$ daily, fluphenazine $2.5 \mathrm{mg}$ daily, benztropine $1 \mathrm{mg}$ daily, and oral analgesics. 
Admission findings included temperature of $40^{\circ} \mathrm{C}$, blood pressure of $118 / 70 \mathrm{mmHg}$, pulse $96 / \mathrm{min}$, and respiration $26 / \mathrm{min}$, a marked hearing deficit, and mild parkinsonism (increased muscle tone and tremor). Blood tests were notable for WBC of $34.5 \times 10^{9} \mathrm{E} / \mathrm{liter}$, increased liver enzymes (lactate dehydrogenase $271 \mathrm{U} /$ liter, alkaline phosphatase $236 \mathrm{U} /$ liter, gamma glutamyl transferase $307 \mathrm{U} /$ liter, aspartate amino-transferase $102 \mathrm{U} /$ liter, and alanine aminotransferase $125 \mathrm{U} /$ liter), and serum CK of $4543 \mathrm{U} /$ liter (with negative MB). Electrocardiogram did not reveal any ischemic changes, and septic work-up (cerebrospinal fluid, blood and urine cultures, and chest X-ray) was unrevealing.

Empiric antibiotic therapy for presumed hepatobiliary infection was initiated. Over the following day, the patient defervesced (temperature $36.6^{\circ} \mathrm{C}$ ), with a marked drop in serum CK (1467 U/liter) and WBC $\left(17.4 \times 10^{9} \mathrm{E} /\right.$ liter $)$. However, a psychiatric consultation was obtained to evaluate for NMS. On mental status examination, the patient was alert, oriented, but unable to communicate effectively because of his hearing deficit. There was no evidence of acute behavioral or psychological abnormality. In the absence of acute psychotic symptoms, discontinuation of fluphenazine was recommended. Over the subsequent four days, the patient continued to improve, and at the time of discharge from the hospital, his serum CK was 398 $\mathrm{U} /$ liter WBC was $7.5 \times 10^{9} \mathrm{E} /$ liter, and liver enzymes were close to normal.

\section{CASE \#3}

A 35-year-old man with acquired immunodeficiency syndrome (AIDS) dementia complex and hypertension entered the hospital for evaluation and treatment of new-onset lethargy, weakness, and peripheral neuropathy. His past psychiatric history was significant for depression and one suicide attempt (medication overdose). Medications on admission included fluconazole, acyclovir, trimethoprim, and dapsone. General physical and neurological examination did not reveal any significant abnormalities. On mental status examination, he exhibited marked anxiety and agitation, with mild cognitive deficits (impaired memory and concentration), but otherwise he was free of psychotic or depressive symptoms. Admission laboratories were remarkable only for a serum CK of $634 \mathrm{U} /$ liter, WBC of $2.5 \times 10^{9} \mathrm{E} /$ liter, and lactate dehydrogenase of $353 \mathrm{U} /$ liter.

Early in the course of hospitalization, the patient's anxiety and agitation worsened, and he became extremely combative and uncooperative with diagnostic procedures. Haloperidol, up to $5 \mathrm{mg}$ daily, was used for sedation, and after receiving a total of $30 \mathrm{mg}$ over six days, his physical and mental condition deteriorated markedly. Temperature increased to $38.0^{\circ} \mathrm{C}$, with fluctuations in blood pressure from $110 / 60 \mathrm{mmHg}$ to $120 / 80 \mathrm{mmHg}$, and pulse rate up to $140 / \mathrm{min}$. The patient also developed lead-pipe rigidity in the extremities, hyperreflexia, and diaphoresis. Organic workup of his symptoms was noncontributory, with negative blood, urine and cerebrospinal fluid studies. Chest X-ray was normal, and head magnetic resonance imaging was remarkable only for mild atrophic changes.

At psychiatric consultation mental status examination revealed confusion, stupor, and impaired speech. Repeated chemistries were remarkable for a serum CK of $501 \mathrm{U} /$ liter and WBC $4.2 \times 10^{9} \mathrm{E} /$ liter. The possibility of NMS was raised, and haloperidol was discontinued, with modest improvement in mental status, autonomic dysfunction, and extrapyramidal symptoms over the next three days. The patient became afebrile with a temperature of $36.5^{\circ} \mathrm{C}$, alert, and was able to follow verbal commands. Two days later, the patient was started on amphotericin B for a presumed fungal infection of the central nervous system, and after five days phenothiazine derivatives were added to his regimen to manage the gastrointestinal side 
effects of the antifungal agent. Subsequently the patient became febrile and stuporous, his motor and autonomic function deteriorated, and serum CK increased to $1161 \mathrm{U} /$ liter.

Follow-up psychiatric consultation, once again, suggested the possibility of NMS and recommended abrupt withdrawal of phenothiazines. Despite the discontinuation of the neuroleptic agent, no significant improvement followed, and the patient was transferred to the intensive care unit and subsequently discharged to an intensive nursing care facility.

\section{DISCUSSION}

First described in the literature by Delay and Deniker (16), as a serious complication of treatment with neuroleptics, NMS is characterized by hyperthermia, parkinsonism, and elevated serum CK. However, retrospective studies have demonstrated that this syndrome is easily confused with other clinical problems in complicated medical patients (17). Our cases illustrate the difficulties of diagnosing NMS in patients with concomitant medical illness.

Diagnosis of NMS in case \# 1 was based on consideration of multiple factors. His risks for developing NMS included receiving intramuscular high potency neuroleptic (haloperidol) and increased doses of trifluperazine in the setting of a pre-existing brain pathology (mental retardation) and other concurrent risk factors (see Table 1). Evaluation of his clinical presentation satisfied both Keck and Caroff's diagnostic criteria of NMS (see Tables 2 and 3). Although fever could have been attributed to the pneumonia, the patient's medical condition could not explain the severe parkinsonism and autonomic instability. Marked resolution of the symptoms following abrupt withdrawal of neuroleptics, and institution of supportive therapy and treatment with bromocriptine, further supported the diagnosis of NMS. Finally, elevated serum CK may have been secondary to bone fracture, but the dramatic drop in CK level following discontinuation of haloperidol and trifluperazine also favored the diagnosis of NMS. It is of note that this patient presented with a history of iron deficiency anemia, which has been reported by some investigators as a potential risk factor for NMS (18).

In contrast to patient \# 1, NMS was ruled out in patient \#2 following consideration of his symptoms, clinical course, and lack of the significant risk factors (see Tables 1-3). The patient had a history of chronic schizophrenia and mental retardation, but the absence of any recent change in the dose or route of administration of fluphenazine made the diagnosis of NMS less likely. The mild extrapyramidal symptoms in this patient, although suggestive of NMS, may have been due to his pre-existing parkinsonism. Furthermore, the course of this patient's illness was not typical for NMS. Recent studies have shown that the mean duration of NMS following appropriate treatment is 6.8 days (19). Thus, the rapid resolution of fever, leukocytosis, and liver enzyme alterations were more likely due to antibiotic therapy for the presumed biliary tract infection, rather the discontinuation of fluphenazine.

The presentation and clinical course of patient \#3 illustrates a diagnostic dilemma. Despite this patient's young age, his concurrent brain pathology (AIDSdementia complex) put him at high risk for NMS when exposed to high potency 
neuroleptics (6). His clinical course is consistent with NMS by both Keck and Caroff's diagnostic criteria (see Tables 2 and 3). Furthermore, the remission and relapse of symptoms after withdrawal of haloperidol and initiation of phenothiazine derivatives respectively, also favor the diagnosis of NMS. However, it is difficult to distinguish the neuroleptic side effects from symptoms of AIDS-dementia complex. Human immunodeficiency virus (HIV) infection has been associated with subacute encephalitis and aseptic meningitis characterized by mental confusion progressing to dementia, fever, metabolic abnormalities, fatigue, and movement disorder (20). Therefore, the hyperthermia, motor dysfunction, and altered sensorium in this patient could be attributed to HIV encephalopathy, especially in the view of the overall progression of the symptoms even after the final withdrawal of phenothiazine derivatives.

These three cases illustrate the difficulties that clinicians face in accurate identification of NMS in the medically ill. Careful review of the temporal relationships of drug administration and the sequence and nature of clinical manifestations as the syndrome evolves, facilitate the diagnosis. Even when diagnostic uncertainty exists, we propose that in high-risk patients, neuroleptic medications should be withheld, and non-neuroleptic treatment strategies be considered to control the residual behavior problems.

\section{ACKNOWLEDGEMENTS}

The authors thank David P. Agle, M.D., for his critical review of this manuscript and helpful comments.

\section{REFERENCES}

1. Delay J, Pichot P, Lemperier T, Elissalde B, Peigne F: Un neuroleptique majeur nonphenothiazine et non-reserpinique, l'haloperidol, dans le traitement des psychoses. Ann Med Psychol 1960; 118:145-152

2. Davis JM, Janicak P, Sakkas P, Hua J: Neuroleptic malignant syndrome: The First 1000 Cases. Biol Psychiatry 1990; 27 (suppl):132A-133A

3. Mann SC, Caroff SN, Lazarus A: Pathogenesis of Neuroleptic malignant syndrome. Psychiatric Annals 1991; 21:175-180

4. Otani K, Horiuchi M, Kondo T, Kaneko S, Fukushima Y: Is the Predisposition to neuroleptic malignant syndrome genetically transmitted? Br J Psychiatry 1991; 158:850 853

5. Parsa MA, Rohr T, Ramirez LF, Meltzer HY: Neuroleptic malignant syndrome without neuroleptics. J Clin Psychopharmacol 1990; 10:437-438

6. Lazarus A: Neuroleptic malignant syndrome and preexisting brain damage. J Neuropsychiatry and Clinical Neurosciences 1992; 4:185-187

7. Keck PE Jr, Pope HG Jr, Cohen BM, McElroy SL, Nierenberg AA: Risk factors for neuroleptic malignant syndrome. Arch Gen Psychiatry 1989; 56:914-918

8. Kline SS, Mauro LS, Scala-Barnett DM, Zick D: Serotonin syndrome versus neuroleptic malignant syndrome as a cause of death. Clinical Pharmacy 1989; 8:510-514

9. Price WA, Zimmer B, Kucas P: Serotonin Syndrome: A Case Report. J Clin Pharmacol $1986 ; 26: 77-78$ 
10. Castillo E, Rubin RT, Holsboer-Trachsler E: Clinical differentiation between lethal catatonia and neuroleptic malignant syndrome. Am J Psychiatry 1989; 146:324-328

11. Guze BH, Baxter LR Jr: Neuroleptic malignant syndrome. N EJ Med 1985; 313:163-166

12. Garoff SN, Mann SC, Lazarus A, Sullivan K, Macfadden W: Neuroleptic malignant syndrome: Diagnostic issues. Psychiatric Annals 1991; 21:130-147

13. Keck PE Jr, Sebastianelli J, Pope HG Jr, McElroy SL: Frequency and presentation of neuroleptic malignant syndrome in a state psychiatric hospital. J Clin Psychiatry 1989; 50:352-355

14. Sakkas P, Davis JM, Hua J, Wang Z: Pharmacotherapy of neuroleptic malignant syndrome. Psychiatric Annals 1991; 21:157-164

15. Caroff SN: Neuroleptic malignant syndrome. J Clin Psychiatry 1980; 41:79-83

16. Delay J, Deniker P: Drug-induced extrapyramidal symptoms. In: Vinken PJ, Bruyn OW, eds. Handbook of Clinical Neurology (6): Diseases of Basal Ganglia. 1968; 248-266

17. Pope HG Jr, Keck PE Jr, McElroy SL: Frequency and presentation of neuroleptic malignant syndrome in a large psychiatric hospital. AM J Psychiatry 1986; 143:1227-1233

18. Gurrera RJ: Serum iron and neuroleptic malignant syndrome. Am J Psychiatry 1991; 148:1405-1406

19. Gelenberg AJ: The Best Treatment for NMS. Biological Therapies in Psychiatry (Newsletter) $1992 ; 15(4): 13$

20. Pajeau AK, Roman GC: HIV Encephalopathy and Dementia. Psychiatric Clinics of North America 1992; 15:455-466 